Accountability in Research, 2000. 8: p. 77. Reprinted with permission of publisher Taylor and Francis.

\title{
TEN YEARS OF COLD FUSION: AN EYE-WITNESS ACCOUNT
}

\author{
F. Scaramuzzi \\ ENEA - Dipartimento Energia, Divisione Fusione, Centro Ricerche Energia \\ C.P. 65 - 00044 Frascati, Rome, Italy
}

\section{1 - INTRODUCTION}

The name of Cold Fusion (CF) comes from the interpretation given to certain phenomena taking place in a metal lattice roughly at room temperature, in terms of nuclear fusion, say between two deuterium nuclei: cold in comparison with the high temperatures of thermonuclear fusion $\left(10^{8}\right.$ K). The first time this was suggested was in the Spring of 1989, ten years ago, by Fleischmann and Pons (1): their experiment gave rise to much turmoil all over the world, ending within a few months with the scientific community rejecting the experiment and thus this interpretation. Research in CF continued nevertheless in a few laboratories, mostly in the USA, Japan, Italy, Russia and China; International Conferences were held regularly, roughly every 1.5 years. However, after ten years, in spite of undeniable (although not overwhelming) progress in the field, there is hardly any communication between this small CF community and the scientific world at large.

I have been active in this field since the beginning, and I have experienced with distress the lack of communication with the rest of the scientific world, mostly because I am aware of the rigorous scientific approach with which the research has been performed by the ENEA Group in which I have been opertating in Frascati. I think that I can contribute in assessing the present situation of CF, both from a scientific and from a 'social' point of view, by acting as a witness of these ten long years.

To start with I will address two of the issues that have been keys to the present lack of communication: the problem of reproducibility and the anomalous nuclear features of the experimental results (2. Some Problems). Then I will try to tell the story of CF as I lived it, which means from my own point of view and in the light of my personal experience: I hope that this report will make clear the logical evolution of the research performed by the ENEA Group of Frascati, resulting in significant progress in the attainment of reproducibility (3. Narrative). I will then try to make a few general statements about the entire field, in order to give some sense of the present state of the art of CF, but without any intention to give an exhaustive review of this discipline, which is not the scope of this Journal (4. State of the art). In the last section I will try to draw some 
conclusions, point out some prospects for the future, and make some comments on communications between the CF community and the rest of the scientific world (5. Conclusions).

\section{2 - SOME PROBLEMS}

I think that two problems, lack of reproducibility and anomalous nuclear features, need to be assessed clearly, because they have been serious concerns of the scientific community, resulting in deep skepticism towards CF. This is what I will try to do in the following.

\section{1 - Reproducibility}

Recently I was watching a TV-show, one of those designed to explain science to the layman. A well known physicist was asked what he thought of CF. His answer was that it was not good science, because of the lack of reproducible experiments. I wrote to him, presenting the following arguments: a) I agree that reproducibility is a "must" in experimental research; b) however, a new field, at its beginning, is often characterized by lack of reproducibility, and it is the task of the scientists operating in that field to understand what is going on, in order to pursue reproduciblity; c) this has been done in the case of CF, making meaningful, even though slow, progress (I sent him a paper of mine (2) in which I had discussed this problem). My letter did not produce any effect, in the sense that he did not change his mind, and went on demanding reproducibility, as if it were an intrinsic characteristics of research and not something that has to be pursued.

In order to clarify the issue, let me try to propose a few statements about reproducibility. First, what does it mean? Consider a simple desk-top experiment. When you perform it, you choose your sample, you work out a procedure (a protocol), and you get your results. It is reproducible if you obtain the same results with the same kind of sample and the same protocol every time you perform your experiment. A further stage of reproducibility consists in describing your experiment in a scientific publication, with the consequence that any other scientist who performs the same experiment, on the basis of that paper, obtains the same results. Now imagine that you perform your experiment, take note as accurately as you can of its parameters (sample and protocol) and when you repeat it you do not get the same results: the experiment is not reproducible! There are two possible explanations: either the first experiment was wrong, or you did not have the same kind of sample, or follow the same protocol. If, by examining your first experiment, you reach the conclusion that the measurement itself was correct and reliable, you have to accept the second explanation. At this point you start a further stage of your research: you try to understand which features were hidden in the choice of the sample and in the protocol, that could have influenced your results without your being aware, and thus you begin what may be a difficult march towards reproducibility. It is not correct to state, as many have done for $\mathrm{CF}$, that non-reproducibility necessarily means a wrong experiment.

An episode that I will now describe will help to illustrate my previous statements: it occurred in 1992 to the ENEA Group of Frascati, which I was leading. We had been working on CF experiments based on gas loading of deuterium in titanium, looking for neutrons and tritium, and eventually we had reached the conclusion that we should move to a different type of experiment: the measurement of excess heat in palladium charged with deuterium in an electrolytic cell with heavy water (substantially the Fleischmann-Pons experiment). In order to build the cathodes, we took the only palladium sheet that was at hand in the laboratory, constructed the electrolytic cell and put it in an accurate calorimeter, and performed the experiment: the first three runs, with three different cathodes taken from the same sheet, and with the same protocol, gave very clear evidence 
of excess heat production, a couple of orders of magnitude larger than the experimental errors (3). At this point, we had used all the palladium existing in the laboratory, and thus we ordered more of it from the same firm that had provided the previous sample, asking for the same commercial characteristics. When the new palladium arrived, we started another series of experiments, none of which gave any sign of excess heat production. So, there we were: we had no doubt about the correctness of the first measurements, but it had been sufficient to change the sample of palladium for the excess heat to disappear, even though, from a commercial point of view, it was the same kind of palladium. This was the beginning of the project that brought the Group to results quite close to total reproducibility in 1996 (4). I will come back to this subject later.

Many research groups in CF have had similar frustrating experiences. And I am sure that history of science is full of examples of this type, in particular when the sample and the protocol are intrinsically complex. I like to quote a sentence that Wolfgang Pauli wrote in 1931 in a letter to Peierls: "One shouldn't work on semiconductors, that is a filthy mess; who knows whether any semiconductors exist" (5). Today we are surrounded by semiconductors, and no one has any doubt about the important contribution of Pauli to the development of physics in this century, but in 1931 he was among those who thought that 'the measurement was wrong'. Conversely, sometimes it happens that, even for complex systems, reproducibility is at hand. This has been the case of high critical temperature $\left(\mathrm{HT}_{\mathrm{c}}\right)$ superconductors: in 1986, when these peculiar ceramic compounds were discovered, in a few months everybody was able to synthesize them and to check that they were superconducting. Ever since, research on $\mathrm{HT}_{c}$ superconductivity has been widely developed, with a trend, and thus a growth, totally different from $\mathrm{CF}$.

The comparison between $\mathrm{HT}_{\mathrm{c}}$ superconductivity and $\mathrm{CF}$, presented first by David Goodstein in the paper that appears also in this issue of AIR (6), is a good guideline to try to analyze the present state of CF. It is my opinion that the lack of reproducibility has played an important role in the skepticism of the scientific community and in the consequent loneliness of the CF community. It is my personal experience that it is quite frustrating and uncomfortable to work for years in such a situation, sustained only by the knowledge (not the belief) that 'the measurement is not wrong'. Physics offers today too many opportunities to perform interesting and advanced research (even though perhaps not as interesting and advanced as CF), leading to more easily reachable rewards, in terms of accepted results, of number of publications, and thus career advancement, to expect scientists to volunteer to perform research in CF. Add to this the substantial a priori discrimination that many important scientific journals have applied to papers related to $\mathrm{CF}$, and the lack of resources, both human and financial, and you have a picture that justifies the slow progress of this field. On the other hand, I have just stated that reproducibility has been 'almost' reached. Why, then, has nothing changed? The episode that I recounted at the beginning of this section is an indirect answer: one often has the feeling that any attempt to restart communications is doomed in advance. One is tempted to say, as Max Planck is supposed to have said: "A new scientific truth does not triumph by convincing its opponents and making them see the light, but rather because its opponents eventually die, and a new generation grows up that is familiar with it".

\section{2 - Anomalous nuclear features}

Research in CF is characterized by a large variety of experiments, from electrolytic cells to gas loading procedures, from the measurement of the excess heat produced to the detection of nuclear ashes of various kinds: neutrons, tritium, ${ }^{4} \mathrm{He}$ and so on. Of course the concept of nuclear ash comes from the conviction that the phenomena under study are nuclear reactions, which is a central point in CF. For the purpose of this section, I will limit myself, without loss of generality, to excess heat experiments, where the large amount of heat produced cannot be accounted for by 
chemical reactions; thus, by default, it is deduced that it comes from nuclear reactions. The most immediate hypothesis in Fleischmann-Pons type experiments is that a fusion reaction between two deuterium nuclei (deuterons) is responsible for the heat production: this is what was proposed in 1989 (1). This reaction has been extensively studied, mostly through experiments performed with the help of particle accelerators, which means in quasi-vacuum and with energetic particles $\left(>10^{5}\right.$ $\mathrm{eV}$ ). This is quite different from the CF experiments, which take place in condensed matter at room temperature (energies of the order of a small fraction of an $\mathrm{eV}$ ). The following scheme shows the well-known rules for the $\mathrm{D}+\mathrm{D}$ fusion reaction:

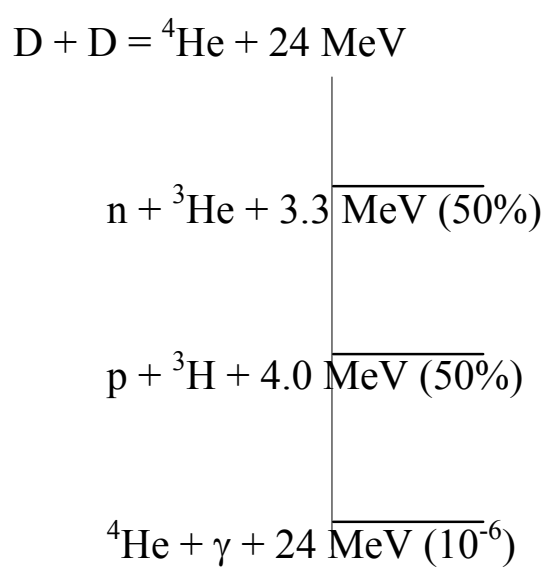

( $\mathrm{n}=$ neutron, $\mathrm{p}=$ proton, in parenthesis the probabilities of the three final branches). The first step in the reaction is the creation of a ${ }^{4} \mathrm{He}$ nucleus with an excess energy of $24 \mathrm{MeV}$. In vacuum this enormous energy explains the high probability for this nucleus to decay into two pieces (the first two branches); only very rarely is the excess energy emitted in a $\gamma$-ray, leaving an unbroken ${ }^{4} \mathrm{He}$ nucleus (third branch). If a D+D reaction is what happens in CF experiments, then these rules are not at all obeyed, because the amount of thermal energy produced would correspond to a tremendous emission of neutrons (just to cite the only particles that go through almost everything), about 5 orders of magnitude higher than the measured flows.

This (and much more) is what I meant by the title 'anomalous nuclear features'. It has been said that, in order to justify D+D fusion reactions in CF experiments with deuterium in palladium, three 'miracles' are necessary. The first one is a dramatic increase of the probability for fusion to take place. If you try to extrapolate to low energies the known probabilities at high energies, you find that the probability of such an event is some 50 or more orders of magnitude lower than that needed to account for the measured excess heat (7): in other words, there is no chance that two deuterons will fuse at room temperature. The second miracle pertains to the absence of neutrons (and tritium, and so on) as the ashes of the reaction. You have to assume that the branching ratio (the relative probabilities of the three branches) is also dramatically altered: you must imagine that the first two reactions become highly improbable, while the third, the one giving rise to a ${ }^{4} \mathrm{He}$ nucleus, has a very high probability, almost $100 \%$. But here we need the third miracle, since we do not see any $\gamma$-rays: thus we have to make the hypothesis that the $24 \mathrm{MeV}$ of excess energy are in some way transformed in heat in the lattice of the host metal, the excess heat that you measure with your calorimeter. If these three miracles are produced, then the only expected ash is ${ }^{4} \mathrm{He}$ : this has been searched for, and in some instances it has been found. But to detect small amounts of ${ }^{4} \mathrm{He}$ is quite difficult, and this is one of the characteristics whose reproducibility is still under investigation. 
Accepting the three miracles does not imply a violation of the fundamental laws of physics: in particular, conservation of mass plus energy is satisfied. One possible solution is to suppose that what makes the difference is the fact that the reaction takes place within condensed matter, rather than in vacuum. However, this is not easy to swallow. It has been said that there is no chance that the lattice, with its long characteristic times $\left(10^{-12} \mathrm{~s}\right)$ and large distances $\left(10^{-10} \mathrm{~m}\right)$ can influence nuclear events, whose typical times are in the order of $10^{-20} \mathrm{~s}$ and whose distances are of about $10^{-15}$ $\mathrm{m}$ : to quote Goodstein (6), "when the nucleus is doing its thing, the atoms of the crystal are far away and frozen in time". But, at this point, a comparison with the Mössbauer effect (which Goodstein also makes) is quite natural. In some nuclei $\left({ }^{191} \mathrm{Ir},{ }^{57} \mathrm{Fe},{ }^{67} \mathrm{Zn}\right)$ the emission of a $\gamma$-ray from a nucleus embedded in a lattice can take place in two entirely different ways, depending on macroscopic properties of the lattice, such as the temperature. In one of the two ways, the emitted $\gamma$ ray has an extremely narrow line-width, coincident with the natural width, which means that the emission takes place as if the recoil were taken up by the entire lattice. To be sure, the energies involved are quite different with respect to $\mathrm{CF}$ (tens of $\mathrm{keV}$ instead of MeV's), and theories explaining the phenomenon have been devised and have been accepted by the scientific world. But then, what strikes me is that, however you look at it, a macroscopic parameter of the condensed matter system, the temperature of the lattice, is responsible for switching the emission of the $\gamma$-ray from one mode (large line-width) to the other (collective behavior of the lattice): this means that the lattice is able to influence the performance of a nuclear event. Giuliano Preparata has attempted a different interpretation of the Mössbauer effect (8) and has proposed a theory for CF (9), both based on the application of Quantum ElectroDynamics (QED) to condensed matter.

These 'anomalous nuclear features' are another reason why the scientific community is very skeptical about CF. Incidentally, even in the CF community there are attempts to interpret the phenomena in terms of (in my opinion unlikely) electromagnetic (i.e., chemical) interactions, which would avoid calling for nuclear events. I think that the experimental evidence of nuclear ashes, including the rather new but impressive field of 'transmutations' (see below), even though not as strong as the evidence for excess heat, is nevertheless quite convincing.

\section{3 - NARRATIVE}

\section{1 - The start-up}

At the end of March 1989, like many scientists around the world, I was very much impressed by the press news from Utah, reporting the experiments of Fleischmann and Pons (1) and of Steven Jones (10). I must confess that I was very skeptical about the excess heat results (how can you imagine D+D fusion without neutrons?), and as a physicist I was reluctant to work in such a 'dirty' stuff as electrolysis. With a very naive reasoning, that I am sure was shared by many others at that time, I thought that a more physical approach, such as gas loading of deuterium in an appropriate metal, should succeed as well in obtaining the fusion of two deuterons. I spoke with the two young physicists of the Group, Antonella De Ninno and Antonio Frattolillo, and with the technicians Peppino Lollobattista, Lorenzo Martinis and Luciano Mori, and we decided to try a simple experiment: charging deuterium in titanium with a gas loading procedure and looking for neutrons while performing temperature cycles. We asked and obtained the participation of experts in neutron detection, Marcello Martone and Salvatore Podda, and performed the experiment: in one week we had two extended periods (tens of hours) with an impressive emission of neutrons. This result was communicated to the management of ENEA, a paper was written (11), a patent was issued, and the 
results were presented in a seminar and in a press conference (the latter seemed to be a common occurence at that time).

I will not go on recounting what happened as a consequence of these first events: this has been described in the paper by David Goodstein quoted above (6). Here I will limit myself to a brief overview of the scientific evolution of our research in the first couple of years. We were soon aware of the very poor reproducibility of our experiment, because we succeeded in repeating the event very seldom over tens of trials. We analyzed our first results many times, wondering whether there was anything wrong, and we could never find any artifact: I am still convinced that those events, even though not reproducible, were real. (Eventually, years later, we learned that another Italian Group had found the same evidence for our second event, following the same procedure (12).) In May 1989 there was a workshop on CF in Santa Fe (NM), organized by the US Department of Energy (DOE) and by the Los Alamos National Laboratory (LANL), and I had the opportunity to contact other groups searching for evidence of neutrons, in particular those of Steven Jones in Provo, Utah, and of Howard Menlove in LANL. As a consequence of the information exchanged, we decided to adopt their sophisticated neutron detectors. The measurements with such a detector were successful, and we presented them at the 1990 March meeting of the American Physical Society in Anheim (CA) and at the first of the international conferences on CF, held in Salt Lake City also in March 1990 (13). The data were rather poor and of much lower intensity than our first ones, but consistent with those obtained by the other groups. The next step was designed to reduce the background: we performed a series of measurements in the Gran Sasso laboratory, a very advanced facility 1600 meters under ground, near L'Aquila, Italy, belonging to INFN (Istituto Nazionale di Fisica Nucleare), where the neutron background is reduced by a factor $10^{3}$. The result was substantially negative: none the less, we reported the results at the Provo meeting in October 1990 (14) and at the second international conference on CF, in Como, Italy, in July of 1991 (15). There had been also theoretical attempts to explain our results not in terms of a real cold fusion, but rather as a hot fusion on a microscopic scale, the energy to justify it being provided either by electromagnetic forces (16) or by the mechanical stress induced by the strong temperature gradients produced during our procedure (17).

Summing up the state of the art of our research activity two years after its beginning, we were not happy at all: on the one hand, our efforts to conquer reproducibility were making no progress, on the other hand the explanation in terms of 'hot fusion on a microscopic scale' rendered the issue much less interesting than we had hoped. Furthermore, I was still very skeptical about the reality of excess heat measurements, considering neutron and tritium measurements the only relevant and reliable evidence. This conviction was shared by many coldfusioners, and this created a division in the field (what Goodstein (6) calls the 'good' and 'bad' kind of CF). The Provo workshop quoted above was dedicated only to experiments based on the detection of nuclear particles, and I participated in its organization, and I appear as one of the editors of the Proceedings (even though my contribution was quite small).

In this state of mind we participated to the Como Conference in July 1991.

\section{2 - The 1991 Como Conference}

I am convinced that the Como Conference was the most important in the short history of CF up to now. What I mean is that in that Conference results were presented that produced a real progress in the field, and influenced the research of the whole community in the following years. I will cite here the three issues that I consider most important: 
Excess heat versus nuclear emissions. There were many confirmations of excess heat measurements in experiments performed in electrolytic cells, even if the amount of the heat produced was quite variable: it was possible to say that there was a first step forward towards reproducibility. Conversely, the measurement of nuclear emissions, in particular neutrons and tritium, even when accurately performed, gave quite random results, far from reproducibility, and at a very low level, often hardly detectable over the background. The attempt to measure neutron energies gave contradictory results, often with no correlation with any theory. Furthermore, it was very difficult to find in a single experiment any correlation between excess heat and nuclear emissions: it seemed that they were totally independent of each other.

Excess heat versus $\mathrm{D} / \mathrm{Pd}$ ratio. One of the most significant results was presented by Mike McKubre et al. (18): at SRI (Stanford Reasearch Institute) they had constructed an accurate calorimeter for electrolytic closed cells, and had also developed methods to measure the amount of deuterium absorbed in the palladium cathode, expressed as $\mathrm{D} / \mathrm{Pd}$ ratio (atomic). This had allowed them to find one of the most important new features of CF: the existence of a threshold of the D/Pd ratio, below which it was not possible to produce excess heat. The value of this threshold, for the experimental configuration of the SRI experiment, was about $0.9-1.0$. A posteriori, this would explain why $\mathrm{CF}$ phenomena were not easy to produce: electrochemists know well that reaching a D/Pd ratio of 0.67 is quite simple, while going beyond this limit is not easy at all. This information had the important result of switching the attention of the experimentalists struggling for reproducibility from the general problem of producing excess heat to the more specific and more controllable problem of overcoming the quoted threshold. It had also an ancillary consequence for me, a physicist totally ignorant of electrochemistry: to understand the main reason why electrolysis is so much more efficient than gas loading in CF experiments. If you look at the phase diagram of the deuteriumpalladium compound you find that in order to obtain high $\mathrm{D} / \mathrm{Pd}$ ratios at room temperature you have to use very high pressures. This is quite impractical in gas loading experiments, and conversely it is unnecssary in electrolysis, where the electrolytic mechanism itself can be considered equivalent to applying very high pressures to the ions entering the metal lattice. It seemed that, until one found a different, more efficient way of loading by gas, electrolysis provided a much better way to reach and overcome the famous threshold.

Another important communication was presented by Melvin Miles of the China Lake laboratory of the US Navy (19). They had collected the gases evolved from an electrolytic cell with which they were performing CF experiments, and had mass-analyzed them with the collaboration of a Group at the University of Texas at Austin, trying to correlate the production of excess heat with the presence of ${ }^{4} \mathrm{He}$ in the gases. The correlation was found and was at least qualitatively consistent with the hypothesis presented above, that ${ }^{4} \mathrm{He}$ is the relevant ash in this type of experiment. It was the first convincing direct evidence that the excess heat is of nuclear origin.

The results just described had a noticeable impact on the development of $\mathrm{CF}$, and in particular they had an immediate effect on the activity of my Group at ENEA Frascati: we decided to switch from the experiments that we had been doing to experiments with electrolytic cells, looking for excess heat. In fact, there was another Group at ENEA Frascati, including Luciano Bertalot, Francesco De Marco, Aurelio La Barbera and Vittorio Violante: they were mostly doing experiments with electrolytic cells, with heavy water and palladium, looking for neutrons and tritium: one of them (ALB) spent a few months at the Texas A\&M University, performing calorimetric measurements on an electrolytic cell, and obtaining clear evidence of excess heat in one run out of three (20). The two Groups merged for this new project. 


\section{3 - Excess heat at ENEA Frascati}

I described in section 2a the episode in which the ENEA Frascati Group saw a clear evidence of excess heat production in 1992 and the ensuing disappointment with the 'new' palladium. Convinced of the reality of excess heat production, the Group went on trying different experimental arrangements, and trying to address issues that could contribute on the one hand to a better comprehension of the observed phenomena and on the other to the achievement of better reproducibility. I will just cite here some of the features that, in my opinion, contributed to progress in the field.

In the first set of measurements (3) the cathode was mounted in such a way that one of its sides was facing the anode, being immersed in the electrolyte, while the other was facing at a sealed volume containing deuterium gas. Monitoring the pressure in this volume gave information on the permeation of deuterium through the cathode. It was found that during the periods of excess heat production deuterium gas flowed from the volume into the metal, the opposite of what happened when there was no excess heat. I do not know what this means, but it is another distinctive feature of this phenomenon.

One of the arguments against the reality of excess heat was the following. Normally it takes a lot of time under electrolysis, sometimes weeks, in order to 'charge' the system, so that it can produce excess heat. One could imagine that there is an unknown mechanism of accumulation of energy (common chemical energy) in the system during this phase. At a certain point this energy is released, and is interpreted as excess heat: if this were true, the phenomenon would be much less interesting, because nuclear reactions wouldn't have to be called for. In two of our experiments we were able to refute this argument. In run n.3 of the first set of experiments (3) the excess heat production started a very short time, minutes, after the beginning of electrolysis and lasted almost 24 hours. In the most spectacular of our results, presented at ICCF5 ( $5^{\text {th }}$ International Conference on Cold Fusion) (21), we had the production of a large amount of excess heat, up to $600 \mathrm{~kJ}$, with a peak power of $11 \mathrm{~W}$ (compared to $8 \mathrm{~W}$ of power input), in the last three days of a 50-day long run. We had performed an accurate calorimetry of the experiment during the whole run. Thus, we could account for all the heat produced, within experimental error $(50 \mathrm{~mW})$. If we imagined that all the experimental error was dedicated to this anomalous accumulation of energy, a very unlikely hypothesis, this still could account for less than one half of the total excess heat measured.

In this same experiment a very suggestive feature appeared. While measuring the balance of the calorimeter, to detect excess heat, we were also monitoring the voltage applied to the cell (through a constant current power supply). In the three periods in which excess heat was produced we found that the measurement of the voltage was characterized by a totally different 'mode', and the 'switch' between the two modes was very abrupt and coincident with the start of excess heat production, as if a transition of phase of the entire thermodynamic system had occurred. The possibility that a phase transition is the basic condensed matter phenomenon that gives rise to CF is quite reasonable: it has been proposed that it could be related to the deuterium ions moving from the octahedral to the tetrahedral sites in the palladium lattice.

\section{4 - The quest for reproducibility}

The quest for reproducibility has been a central theme of the Group, both because of its intrinsic importance, and because we are aware of the 'social' consequences of its lack: the unavoidable 'loneliness' with respect to the traditional scientific community, which I discussed above. The importance of having a high value of the $\mathrm{D} / \mathrm{Pd}$ ratio, which we had learned at the Como 
Conference, became more and more evident as time went on and thus the episode of the 'good' and 'bad' palladium could be interpreted in terms of different degree of difficulty in pursuing this objective for different 'kinds' of palladium. This was a clear indication that studying the characteristics of palladium and their relation with the deuterium absorption dynamics was the main route to follow in order to obtain high $\mathrm{D} / \mathrm{Pd}$ ratios. Eventually the work done on this problem by the young scientists of the Group (Antonella De Ninno, Aurelio La Barbera and Vittorio Violante) yielded important insight into the main reasons for the difficulty of attaining high loading ratios (22). As a consequence of these insights, they proposed a protocol for the preparation of the sample, and a particular procedure for the first phase of deuterium loading, which eventually allowed the Group to reach a good level of reproducibility: 5 samples out of 6 that had undergone the whole procedure showed very clear excess heat production (4). At this point an intense investment of time and effort was called for, to take advantage of this progress. Unfortunately, what has happened instead in the last few years is that the Group has been reduced from five full-time-equivalent scientists to a staff of two.

I want to mention here another technique to study the dynamics of deuterium in palladium, which was applied in experiments of the Group done in cooperation with another laboratory in the Frascati area (Istituto di Struttura della Materia of the Consiglio Nazionale delle Ricerche): an Xray diffractometer was used to measure in real time the unit cell parameters during electrolysis, thus observing possible changes in the palladium lattice when high values of loading ratio were attained. This technique worked well (23), but we never reached $\mathrm{D} / \mathrm{Pd}$ ratios higher than 0.75 in these experiments. This is a very promising technique that should be pursued in a more systematic way. Unfortunately, this was not possible in our case: the experiment was very 'expensive', in terms of time and work, and the Group was quite small.

\section{4 - STATE OF THE ART}

\section{1 - Science}

Evaluating research in $\mathrm{CF}$ is quite difficult, due to the great variety of experiments and of theories that have appeared in this field: I stopped counting publications when the number passed a thousand a few years ago. It is also difficult because, in the absence of normal relations with the traditional world of science, within the CF community there has been too little criticism, and, I would say, a lack of serious refereeing. Thus the papers are not all of good quality, and their results are sometimes questionable. Nevertheless, there are many good experiments and corresponding good papers: there is no doubt that there has been progress in research on CF. Edmund Storms has tried twice to review it, producing two very detailed papers, complete with very extended bibliographies (24): I recommend them to those who want to have detailed knowledge of this field. Here, both because I do not feel able to perform such an enterprise satisfactorily, and because this is beyond the purpose of this article (and of this Journal), I will try to give a short summary of the most important areas: once again, it will reflect my personal view on the field, which does not pretend to be exhaustive.

Let me start with the effort to attain reproducibility. I want to mention here the work of Giuliano Mengoli, in Padua, in which excess heat measurements in electrolytic cells have been performed in different configurations, while working at temperatures close to the boiling point of water. The results are quite reproducible (25). (Experiments at 'high' temperatures had already been performed by Fleischmann and Pons, who showed also an interesting feature, called "heat after death", i.e., the persistence of heat production after all the electrolyte was evaporated (26).) Another interesting 
method worth mentioning is the one followed in the experiments performed by Giuliano Preparata et al. The idea is that the presence of an electric potential in the palladium cathode can influence its chemical potential (27), with the effect of allowing higher $\mathrm{D} / \mathrm{Pd}$ ratios: in this way, excess heat is more easily obtained, without worrying too much about the 'material science' characteristics of the metal (the idea arises out of Preparata's theory cited above (9)). In order to have high enough potentials, the cathode is a long thin Pd-wire, with a voltage applied at its ends: the results are quite reproducible (28). The route chosen by Francesco Celani, consisting of coating the cathode with appropriate materials in order to prevent the deuterium from escaping is also quite promising (29).

There are a number of experiments that seem to show that it is possible to have excess heat production in a different experimental system: nickel and hydrogen rather than palladium and deuterium. In this case the only reasonable explanation for nuclear fusion reactions is the fusion of hydrogen with one of the few deuterons that are always present in hydrogen as impurities. This idea is reminiscent of the work of Julian Schwinger, who examined the possibility of such a reaction as a general explanation of CF (30). Excess heat has been detected in light water electrolysis experiments $(25,31)$, in electrolysis in so-called 'Patterson cells', in which the cathode is constituted by thousands of small plastic spheres coated with layers of palladium and nickel all packed together (32), and in gas loading experiments (33).

There has been a growing interest in the detection of 'nuclear ashes'. A number of careful and refined experiments have been reported that are designed to detect 'traditional' ashes, such as neutrons, tritium, and so on, and to their correlation with excess heat: with particular mention is the work of the Osaka Group led by Akito Takahashi (34). There has been an increasing effort to detect ${ }^{4} \mathrm{He}$, which is an intrinsically difficult task. The results have been interesting, but the reproducibility has been far from satisfactory: I want to note here a very clear signal found by the Turin Group in a gas loading experiment (35) and an elaborate but convincing result by Daniele Gozzi, in which the balance between energy produced and number of ${ }^{4} \mathrm{He}$ atoms is consistent with the model of $\mathrm{D}+\mathrm{D}$ fusion (36). A totally new line of work, known under the name of "transmutations" has been developed in the last few years. Here, in experiments of various types, stable isotopes that were absent at the beginning of the experiment are found. This seems to indicate nuclear reactions other than $\mathrm{D}+\mathrm{D}$ fusion, which is very difficult to understand. There is a wide spectrum of publications on this field, of which I cite just a few representative papers due to the University of Illinois Group led by George Miley (37) and to the Sapporo Group led by Tadahiko Mizuno (38); this field seems to be expanding, as evidenced in the last conference, ICCF7, held in Vancouver, Canada, in April of 1998.

Much could be said about theories: many and extremely various ideas have been presented. But, once again, I will not try to describe them. Let me just make a couple of general observations. The traditional recurrent mechanism that governs the development of science, alternating theory and experiment, so that they can check each other, is hardly possible if you are still struggling with the lack of reproducibility: this problem has seriously hampered the development of theories. The second observation is that, accepting the nuclear nature of the phenomena of CF, as I do, and considering the arguments discussed in section $2 \mathrm{~b}$ about the 'anomalous nuclear features' of CF, it is not possible to explain CF on the basis of two-body interactions. It is necessary to demand the existence of a collective and coherent mechanism governing the phenomena. Such a mechanism was proposed immediately by Preparata et al (9) and others, of which I note here Scott and Talbot Chubb (39), have been proposed since then. 


\section{2 - The CF community: science versus utility}

A little history is useful in order to better understand the present status of the CF community. As I said at the beginning, towards the end of 1989 there was a rejection of CF by the traditional scientific community, leaving behind a number of ongoing efforts with a particular geographic distribution. In the USA there were many groups operating in spite of the lack of dedicated funds by DOE and NSF. In Japan there was considerable interest from the beginning. Within Europe there was continuing research in a number of groups in Italy, plus a small Group in Spain. China and India were also active, and in due time Russia also showed up. Most of these initiatives were due to small groups, with little or even no funding. Its activity is witnessed by the periodic International Conferences that have been held since then, 8 of them up to now. But there were also three big initiatives that were launched in the years following 1989, that I want to note here:

The Electric Power Research Institute (EPRI) made an important investment in CF research, initially in a number of areas, eventually mostly in excess heat experiments with $\mathrm{D} / \mathrm{Pd}$ systems, that were performed at the Stanford Research Institute (SRI). This project was active for many years, and was terminated in 1995.

There was an important early project conducted by IMRA, an institution tied to the Japanese industry Toyota (in fact, this was a personal decision of one of the heads of this leading group, Minoru Toyota): three laboratories were created, two of them in Japan (in Sapporo and in Nagoya), and one in Europe, at Sophia Antipolis, near Cannes (where eventually Fleischmann and Pons became active). This project too has been terminated quite recently.

Another important Japanese initiative was taken by the Ministry for International Trade and Industry (MITI) with an additional contribution from a consortium of industries. The project involved a specialized laboratory built for the purpose, and the collaboration of Universities on more fundamental aspects. This project was terminated in 1998.

One could be tempted to interpret the closure of these three important projects as a demonstration that $\mathrm{CF}$ research is failing in its objective to become a well defined discipline in science. I am personally convinced that this interpretation is definitely wrong. Let me explain why. One of the common characteristics of these projects is that they were promoted by agencies (in a general sense) that were highly interested in the potential energetic applications of CF (it is undeniable that the picture that I sketched earlier indicates the possibility to produce particularly clean nuclear energy, which is something mankind has been dreaming for decades). Thus, their expectation was to be able to develop practical applications of CF in a few years. From the description of the progress in science that I have reported above it is clear that, despite indubitable scientific realities, progress in its development has been quite slow, both because of the intrinsic difficulties of the field, and of the very scarce resources that have been dedicated to its study. We are still far from developing applications. Thus, it was to be expected that enterprises that were born with the aim of having a practical fall-out in short time had to give up. I am still convinced that a lot of basic research is needed, in order to better understand the science underlying CF, before practical objectives can be seriously addressed: this can be better pursued by small groups that proceed with this clear idea in mind.

And this is in my opinion what is beginning to happen. Let me mention a few events that show this tendency: it could be (and I hope it is) incomplete, but nevertheless it gives the sense that $\mathrm{CF}$ is finally beginning to reenter the scientific world. 
At Grenoble, in France, there is a new laboratory funded by the French CEA (Commisariat a l'Energie Atomique) and by the Grenoble Institut Polytechnique, which has started a research project in $\mathrm{CF}$

The SRI Group, that was funded by EPRI for many years, and then by the Japanese MITI for a couple of years, is presently active in CF research with funds from the US agency DARPA (Defense Advanced Research Projects Agency)

In Italy a new initiative is starting now: a cooperation between ENEA, INFN and LEDA that will allow the creation of a new laboratory at ENEA Frascati with a research program on CF, funded by the Italian Government, for the next three or more years.

Let me mention, to finish, that at the American Physical Society March Meeting of 1999 (the Centennial Meeting) there will be a session, entitled "Palladium Electrochemistry", where papers on $\mathrm{CF}$ will be presented.

\section{5 - CONCLUSIONS}

It has been a long story; let me try to conclude by pointing out some meaningful aspects of this strange adventure of $\mathrm{CF}$, relative to the past, to the present and to the future.

\section{1 - Scientific realities}

There is still no effective dialogue between the CF community and the traditional scientific world. There are extremes on both sides: some claim that CF does not exist, others are convinced that we have already solved the energy problems of mankind. As this paper makes clear, it is my conviction that some of the phenomena known with the name of CF are real, in particular the production of excess heat and its nuclear origin. I am also convinced that it is a very complex matter, that it requires a stronger and longer effort in research, just to understand better the basic phenomena, and that it is too early to consider practical applications. Reproducibility is still an important issue, but much progress has been made, and I think that it is at hand.

\section{2 - Sociology}

A reasonable question is the following: How is it possible that after 10 years the extreme positions about CF have not softened, and nevertheless there is a small community (hundreds of scientists) that goes working on with enthusiasm, in spite of the great difficulty that the general skepticism produces? It seems to me that this question has two different answers. The first is connected to the extreme appeal that the theme of CF has on people. There is the hope of solving one of the most serious problems of the world, the problem of energy, and succeeding in the task means pride, honors, money and so on: even if it is very difficult, even if the chances of succeeding are very small, some think that it is worth pursuing this objective. The other answer is that, no matter how you judge the CF community, there are results that are real, for example, the excess heat and the nuclear ashes do exist, in spite of the lack of reproducibility and of all the difficulties that I have tried to describe in this article. If they were not real, the field would have been abandoned many years ago. Add to this that those who started working on it and got positive results believe in the reality of their results and are willing to go on until a better comprehension of the phenomena is acquired, and some practical application is at hand. It is certainly an unusual situation, one that has never happened in the past. 


\section{3 - The role of chance}

I have often wondered about the influence of chance on my personal history. Consider the first episode: we try an experiment that is very naive, we perform it and get tremendous results, twice in a week. This produces success, excitement, commitment and so on. In two years we realize that it is one of the most irreproducible experiments. Thus, the question comes to the mind: if in our first attempts we hadn't had any neutron emission, what would have we done? The answer is clear to me: we would have abandoned the field after a few more negative attempts, exactly as many others have done, and we would not be involved in research on CF. Now, a posteriori, we know that the probability of seeing neutrons in that experiment was very small: thus, chance was the determinant factor in our participating in the CF adventure. Once again, consider the impasse in which we were at mid-1991: we were disappointed in the lack of reproducibility in our gas loading experiments, we decided to move to excess heat experiments, we did our first experiments with the 'good' palladium, and saw without any doubt the production of excess heat. Suppose instead that we had started with the 'bad' palladium and saw no excess heat: we would have been thoroughly convinced of the non-existence of excess heat and most probably we would have abandoned the field. Once again chance has been the determining factor in our continuing our activity.

\section{4 - The future}

It is evident that this field requires a more massive effort in order to attain steady progress. Propagation of the research into the rest of the scientific community would greatly contribute to this result. As I said before, I have the feeling that this is beginning to happen, and I am optimistic about future developments. As far as the fields of investigation are concerned, I think that the most recent results indicate that there are three important areas to be pursued: systems that yield excess heat without electrolysis, work at temperatures (at least slightly) higher than room temperature, and work with low dimensionality systems (powders, wires, films).

The reasons 'why' one should devote a more massive effort to develop CF are clear to me, and I hope that they are convincing to you, now that you are reading this last paragraph. The study of collective and coherent phenomena promises very high intellectual rewards, and the hope of contributing to the solution of the problem of the energy supply for mankind is highly inspiring. Last but not least, the challenge of studying a field which is not well understood is definitely fascinating. Let me end by quoting Albert Einstein in a sentence that I read during a visit to the Space Museum of Washington: "The most beautiful experience we can have is the mysterious. It is the fundamental emotion which stands at the cradle of true art and true science". 


\section{BIBLIOGRAPHY}

The "International Conferences on Cold Fusion", of the series ICCFn, starting from ICCF3, will be often quoted in this bibliography. Hereafter are their references: in the rest of the bibliography they will be quoted just by the acronym.

ICCF3 "Frontiers of Cold Fusion", Proceedings of the Third International Conference on Cold Fusion, Nagoya, Japan, October 21 - 25, 1992, editor H. Ikegami, published by Universal Academy Press, Inc., Tokyo (1993)

ICCF4: Proceedings of the Fourth International Conference on Cold Fusion, Lahaina, Maui, Hawaii, USA, December 6 - 9, 1993; published in Transactions of Fusion Technology, a Journal of the American Nuclear Society, Vol. 26, N. 4T (December 1994).

ICCF5: Proceedings of the 5th International Conference on Cold Fusion, Monte Carlo, Monaco, April 9 - 13, 1995, published by International Conference on Cold Fusion 5, Valbonne, France (1995).

ICCF6: "Progress in New Hydrogen Energy", Proceedings of the Sixth International Conference on Cold Fusion, Hokkaido, Japan, 13 - 19, 1996, published by "New Energy and Industrial

Development Organization, The Institute of Applied Energy", 1997

ICCF7: Proceedings of the Seventh International Conference on Cold Fusion, Vancouver, Canada, April 19 - 24, 1998, copyright by ENECO, inc., editor F. Jaeger (1998)

1. M. Fleischmann, S. Pons, "Electrochemically Induced Nuclear Fusion of Deuterium", $J$. Electroanal. Chem., 261, 301 (1989); see also M. Fleischmann, S. Pons, Errata, J. Electroanal. Chem., 263, 197 (1989).

2. F. Scaramuzzi, "La fusione fredda otto anni dopo", Energia Ambiente e Innovazione; ENEA Journal, 1/97, 29 (1997)

3. L. Bertalot, F. De Marco, A. De Ninno, A. La Barbera, F. Scaramuzzi, A. Violante, P. Zeppa, "Study of Deuterium Charging in Palladium by the Electrolysis of Heavy Water: Search for Excess heat and Nuclear Ashes", (ICCF3), 365 (1993); L. Bertalot, F. De Marco, A. De Ninno, A. La Barbera, F. Scaramuzzi, A. Violante, P. Zeppa, "Study of deuterium charging in palladium by the electrolysis of heavy water: excess heat production", Il Nuovo Cimento, 15 D, 1435 (1993)

4. F. De Marco, A. De Ninno, A. Frattolillo, A. La Barbera, F. Scaramuzzi, V. Violante, "Progress Report on the Research Activities on Cold Fusion at ENEA Frascati", (ICCF6), Vol.1, 145 (1997)

5. L. Hoddeson, E. Braun, J. Teichmann, S. Weart, Out of the Crystal Maze, Oxford University Press, 121 (1992)

6. D.L. Goodstein, "Whatever Happened to Cold Fusion?", The American Scholar, 63, 527 (1994); printed also in Engineering and Science, autumn 1994, 14 (1994)

7. A.J. Leggett, G. Baym, "Exact Upper Bond on Barrier Penetration Probabilities in Many-Body Systems: Application to Cold Fusion", Phys. Rev. Lett., 63, 191 (1989)

8. G. Preparata, "The Mössbauer effect: a glimpse of the stunning QED coherence of matter", ICAME-95, Conference Proceedings, editor I. Ortalli, Editrice Compositori, Bologna, 955 (1995)

9. T. Bressani, E. Del Giudice, G. Preparata, "First Steps Toward an Understanding of 'Cold' Nuclear Fusion", Il Nuovo Cimento, 101A, 845 (1989).

10. S.E. Jones, E.P. Palmer, J.B. Czirr, D.L. Decker, G.L. Jensen, J.M. Thorne, S.F. Taylor, J. Rafelski, "Observation of cold nuclear fusion in condensed matter", Nature, 338, 737 (1989). 
11. A. De Ninno, A. Frattolillo, G. Lollobattista, L. Martinis, M. Martone, L. Mori, S. Podda, F. Scaramuzzi, "Emission of Neutrons as a Consequence of Titanium-Deuterium Interaction", Il Nuovo Cimento, note brevi, 101, 841 (1989); same authors, "Evidence of Emission of Neutrons from a Titanium-Deuterium System", Europhysics Letters, 9 (3), 221 (1989).

12. M. Fabrizio, C. Manduchi, G. Mengoli, E. Milli, G. Zannoni, "Emissione di neutroni dall'interazione di deuterio gassoso con titanio e con leghe a base di palladio", Proceedings of the Rome Workshop on the status of cold fusion in Italy, University of Rome III, February 1316, 1993, editor B. Stella, 74 (1993)

13. F. D'Amato, A. De Ninno, F. Lanza, C. Pontorieri, F. Scaramuzzi, P. Zeppa, "Search for Nuclear Phenomena by the Interaction between Titanium and Deuterium", Proceedings of the First Annual Conference on Cold Fusion, March 28-31, 1990, Salt Lake City, Utah, USA, sponsored by the National Cold Fusion Institute, 170 (1990)

14. A. De Ninno, C. Pontorieri, F. Scaramuzzi, P. Zeppa, "Emission of Neutron Bursts from a Titanium-Deuterium Gas System in a High-Efficiency Low-Background Experimental Setup", Conference on Anomalous Nuclear Effects in Deuterium/Solid Systems, Provo, Utah, USA, 1990, Proceedings published by American Institute of Physics (Conference Proceedings 228), editors S.E. Jones, F. Scaramuzzi, D. Worledge, 122 (1991)

15. A. De Ninno, A. Frattolillo, F. Lanza, S. Migliori, C. Pontorieri, S. Scaglione, F. Scaramuzzi, P. Zeppa”, The Science of Cold Fusion, Proceedings of the II Annual Conference on Cold Fusion, Como, June 29-July 4, 1991, editors T. Bressani, E. Del Giudice, G. Preparata, published by the Società Italiana di Fisica, Conference proceedings n.33, 129 (1991)

16. S.E. Segre, S. Atzeni, S. Briguglio, F. Romanelli, "A Mechanism for Neutron Emission from Deuterium Trapped in Metals", Europhysics Letters, 11, 201 (1990)

17. E. Tabet, A. Tenenbaum, "Displacement flow and deuteron drag in a metal: a path towards cold fusion", Understanding Cold Fusion Phenomena, edited by R.A. Ricci, F. De Marco, E. Sindoni, Conference Proceedings (Varenna, 15-16 September 1989), Volume 24, Italian Physical Society, 175 (1990)

18. M.C.H. McKubre, R. Rocha-Filho, S.I. Smedley, F.L. Tanzella, S. Crouch-Baker, T.O. Passell, J. Santucci, "Isothermal flow calorimetric investigations of the D/Pd system", The Science of Cold Fusion, Proceedings of the II Annual Conference on Cold Fusion, Como, June 29-July 4, 1991, editors T. Bressani, E. Del Giudice, G. Preparata, published by the Società Italiana di Fisica, Conference Proceedings n. 33, 419 (1991)

19. M.H.Miles, B.F. Bush, G.S. Ostrom, J.J. Lagowski, "Heat and Helium Production in Cold Fusion Experiments", The Science of Cold Fusion, Proceedings of the II Annual Conference on Cold Fusion, Como, June 29-July 4, 1991, editors T. Bressani, E. Del Giudice, G. Preparata, published by the Società Italiana di Fisica, Conference Proceedings n. 33, 363 (1991)

20. L. Bertalot, L. Bettinali, F. De Marco, V. Violante, P. De Logu, T. Dikonimos Makris, A. La Barbera, "Analysis of tritium and heat excess in electrochemical cells with Pd cathodes", The Science of Cold Fusion, Proceedings of the II Annual Conference on Cold Fusion, Como, June 29-July 4, 1991, editors T. Bressani, E. Del Giudice, G. Preparata, published by the Società Italiana di Fisica, Conference Proceedings n. 33, 3 (1991)

21. L. Bertalot, A. De Ninno, F. De Marco, A. La Barbera, F. Scaramuzzi, V. Violante, "Power Excess Production in Electrolysis Experiments at ENEA Frascati", (ICCF5), 34 (1995)

22. A. De Ninno, A. La Barbera, V. Violante, "Selection of Palladium metallurgical parameters to achieve very high loading ratios", (ICCF6), Vol.1, 192 (1997); A. De Ninno, A. La Barbera, V. Violante, "Deformations Induced by High Loading Ratios in Palladium Deuterium Compounds", Journal of Alloys and Compounds, 253, 181 (1997); A. De Ninno, A. La Barbera, V. Violante, "Consequences of lattice expansive strain gradients on Hydrogen loading in Palladium", Phys. Rev. B, 56, 2417 (1997); A. De Ninno, M. Vittori Antisari, C. Giangiordano, "Material science studies aimed at improving the reproducibility of the heat excess experiments", (ICCF7), 103 (1998) 
23. L. Bertalot, F. De Marco, A. De Ninno, R. Felici, A. La Barbera, F. Scaramuzzi, V. Violante, "Deuterium charging in palladium by the electrolysis of heavy water: measurement of the lattice parameter", (ICCF4), 122 (1994); R. Felici, L. Bertalot, A. De Ninno, A. La Barbera, V. Violante, "In situ measurement of the deuterium (hydrogen) charging of a palladium electrode during electrolysis by energy dispersive x-ray radiation", Rev. Sci. Instrum., 66, 3344 (1995)

24. E. Storms, "Review of Experimental Observations about the Cold Fusion Effect", Fusion Technology, 20, 433 (1991); "Critical Review of the Cold Fusion Effect, Scientific Exploration, $10,186(1996)$

25. G. Mengoli, M. Bernardini, C. Manduchi, G. Zannoni, "Calorimetry close to the boiling temperature of the $\mathrm{D}_{2} \mathrm{O} / \mathrm{Pd}$ electrolytic system", J. Electroanal. Chem., 444, 1255 (1998)

26. S. Pons, M. Fleischmann, "Heat After Death", (ICCF4), 87 (1994)

27. Preparata, private communication

28. G. Preparata, M. Scorletti, M. Verpelli, "Isoperibolic Calorimetry on Modified FleischmannPons Cells, J. Electroanal. Chem., 411, 9 (1996)

29. Celani, private communication

30. J. Schwinger: "Nuclear Energy in an Atomic Lattice", Salt Lake City '90, 130 (1990); "Nuclear Energy in an Atomic Lattice. 1", Z. Phys. D, 15, 221 (1990); "Cold Fusion: A Hypothesis", Z. Naturforsch., 45a, 756 (1990); "Cold Fusion: Does It Have a Future?", Evolutional Trends of Physical Sciences, Springer-Verlag, Germania (1991); "Phonon Representations", Proc. Nat. Acad. Sci., 87, 6983 (1990); "Phonon Dynamics", Proc. Nat. Acad. Sci., 87, 8370 (1990); "Nuclear Energy in an Atomic Lattice - Casual Order", Prog. Theor. Phys., 85, 711 (1991); "Cold Fusion Theory - A Brief Hystory of Mine", ICCF4, xiii (1994).

31. There are many papers on this subject; a couple of them are quoted here: R. Notoya, M. Enyo, "Excess Heat Production during Electrolysis of $\mathrm{H}_{2} \mathrm{O}$ on Ni, Au, Ag and $\mathrm{Sn}$ Electrodes in Alkaline Media", (ICCF3), 421 (1993); R.T. Bush, R.D. Eagleton, "Calorimetric Studies for Several Light Water Electrolytic Cells With Nickel Fibrex Cathodes and Electrolytes with Alkali Salts of Potassium, Rubidium and Cesium", (ICCF4), 13 (1994).

32. D. Cravens, "Flowing Electrolyte Calorimetry", (ICCF5), 79 (1996)

33. S. Focardi, R. Habel, F. Piantelli, "Anomalous Heat Production in Ni-H Systems", Il Nuovo Cimento, note brevi, 107 A, 163 (1994).

34. A. Takahashi, "Results of experimental studies on excess heat vs. nuclear products correlation and conceivable reaction model", (ICCF8), 378 (1998)

35. E. Botta, T. Bressani, D. Calvo, C. Fanara, F. Iazzi, "Measurements on ${ }^{4} H e$ Production from $\mathrm{D}_{2}$ gas-loaded Pd Sample", (ICCF6), Vol. 1, 29 (1997)

36. D. Gozzi, F. Celluci, P.L. Cignini, G. Gigli, M. Tomellini, E. Cisbani, S. Frullani, G.M. Urciuoli, "X-ray, heat excess and 4He in the D/Pd system", J. Electroanal. Chem., 435, 113 (1997); erratum: J. Electroanal. Chem, 452, 251 (1998)

37. G.H. Miley, G. Narne, M.J. Williams, J.A. Patterson, J. Nix, D. Cravens, H. Hora, "Quantitative Observation of Tansmutation Poducts Occurring in Thin-Film coated Microspheres during Electrolysis", (ICCF6), Vol.2, 629 (1997)

38. T. Mizuno, T. Ohmori, T. Akimoto, K. Kurokawa, M. Kilaichi, K. Inoda, K. Azumi, S.Shimokawa, M. Enyo, "Isotopic Distribution for the Elements Evolved in Palladium Cathode After Electrolysis in $\mathrm{D}_{2} \mathrm{O}$ Solution", (ICCF6),Vol. 2, 665 (1997); T. Ohmori, T. Mizuno, M. Enyo, "Production of Heavy Metal Elements and the Anomalous Surface Structure of the Electrode Produced During the Light Water Electrolysis on Au Electrode", (ICCF6), Vol. 2, 670 (1997)

39. S.R. Chubb, T.A. Chubb, "Periodic order, symmetry, and coherence in cold fusion", (ICCF7), 73 (1998) 